\title{
Cloud-Native Approach: Educational ICT Infrastructure
}

\author{
Ming-Hsun Yang, Shian-Hua Lin* \\ Department of Computer Science and Information Science, National Chi Nane University, Puli, Taiwan. \\ * Corresponding author. Tel.: +886 49 2915225; email: shlin@ncnu.edu.tw \\ Manuscript submitted November 1, 2016; accepted March 9, 2017. \\ doi: 10.17706/ijeeee.2017.7.2.79-84
}

\begin{abstract}
In the cloud generation, most application services have been moved from the desktop to the cloud, but we still need a desktop environment to operate applications. Privacy, security, geography, and legacy issues such as the inability to migrate traditional applications, desktop maintenance remains an unavoidable topic for cloud-based information infrastructures. We proposed a virtual disk layering and mass deployment mechanism originate from native OS functions. With it, different pre-configured environment OS and application, disk layering as virtual appliances images can run on a hypervisor computer, multicast deploy from 1 to $\mathrm{N}$ machines. In this paper, we use the concept of Cloud-Native to elaborate the management and technology theme of the mechanism.
\end{abstract}

Key words: Virtual disk layering, mass deployment, cloud-native.

\section{Introduction}

In the era of cloud services, x86-based PC is still the dominant desktops. Numerous IT requirements for multi-OS platforms, software installations of commercial and open source applications, backups and disaster recovery are frequently needed to be deployed on demands. However, current large-scale deployment and management solutions are tedious and costly for IT staffs.

In 1999, all schools in Taiwan were connected to the Internet and at least have one computer room. The Internet connections are upgrading to 100MB fiber optic about 2009. A three-tier network structure that includes schools, the City \& County Network Center (CNC), the Regional Network Center (RNC), and connection to backbone form of the Taiwan Academic Network (TANet). The Education Network Center of Chiayi County (ENCCYC) is one of CNC between schools and Yunlin and Chiayi Regional Network Center (YCRNC at CCU), in charge of most educational IT affairs and ICT infrastructure in Chiayi County. ENCCYC is established in response to digital campus infrastructure, wired and wireless Internet connections, and differentiation for ICT educational purposes. To continuous improve above works, we develop a native virtual image layering and mass deploy mechanism.

The structure of the proposed mechanism consists of GRUB4DOS, desktop layering, WinPE with utilities and scripts (see Fig. 1). The computer boots the operating system: After the hardware is booted, the system will do BIOS checking or loading UEFI, load bootloader, then loading installed OS. Thus, let boot loader loads layering virtualized image files as the environment instead of installing OS.

These following procedures explain the future of mechanism:

1) Cross-platform and maintain propose environment can be hosted as a menu: booting multiple operating systems like Windows, Linux, Android-x86, and WinPE.

2) Manage disk image as files: The desktop environment is virtualized into bootable files with native OS 
supports. Applications installed by layering images. Compress these files as a single packed kit, then distribute the kit from network backbone to remote deployment.

3) Disaster recovery: Backup differencing disks, loop files and batch script for renewing these files made environment recovered.

4) From 1 to $\mathrm{N}$ deployment: For system rescue or renew deployment, a light-weight execution environment, associated with networking ability, used for system start-up through the network. With it, single computer environment can be massively duplicated to multiple networked computers.
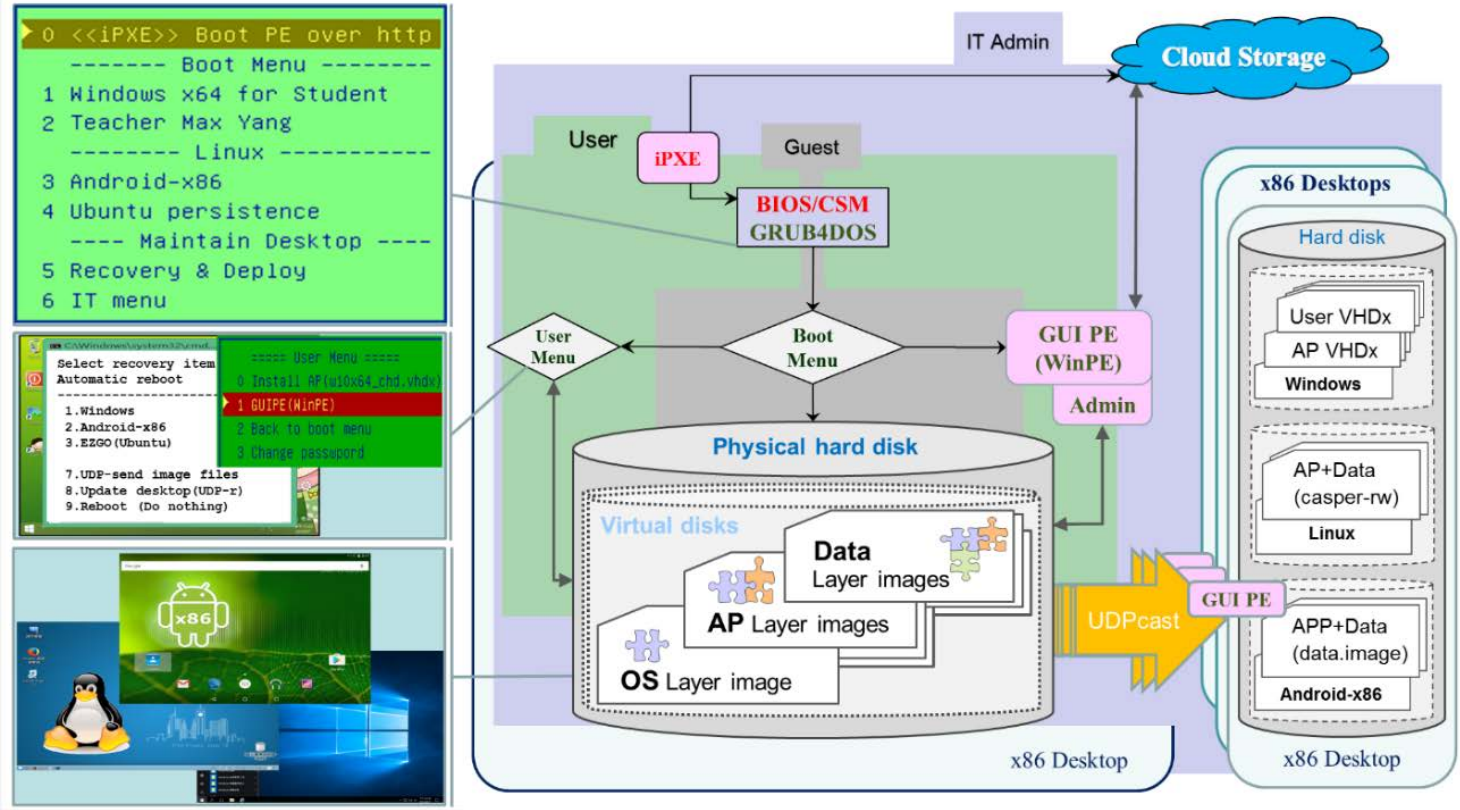

Fig. 1. Mechanism workflow diagram.

The following chapters, we use the concept of Cloud-Native to elaborate the mechanism regarding technology and management.

\section{The Management Themes of the Mechanism}

\subsection{Dev0ps}

DevOps is a set of methods, and architectures for communication, collaboration, and integration among software development, operations, and quality assurance. That is comprehension of the interdependence between development and operation. In the past, most ICT infrastructure of education in Taiwan are traditional x86 based PC, replacement cycle up to 4-8 years, and maintain environment cycle several times a year. By using this mechanism, DevOps, continuous delivery and efficient management made infrastructure agile. The package kit developer updates monthly and renews in cloud storage. The school maintained deploys PC rooms within hours and the computers of education corners within days.

\subsection{Continuous Delivery}

This future is to improve the further work of the mechanism continuously:

- Automate the building, integration, testing and deployment of software at all levels.

- Enhance cooperation in development, testing, and operations personnel.

- Implement an effective configuration management strategy, remain decentralized characteristics.

- Version controls with continuous deployment and zero downtime.

- Manage infrastructure, information, components and dependencies. 
It is made Infrastructure as code, model driven automation, continuous deployment.

\section{The Technology Themes of the Mechanism}

\subsection{Microservices}

The microservice is a single application developed as a set of servlets, each of them runs in its process and using lightweight communication. An independent application named Kazilla of the mechanism; combined administrator mode, fast recovery, and multicast services makes up the system. Take some microservice as example:

1) Disk pre-configure service: An HTTP service made for users to select installation components and generate disk configure kit (see Fig. 2).

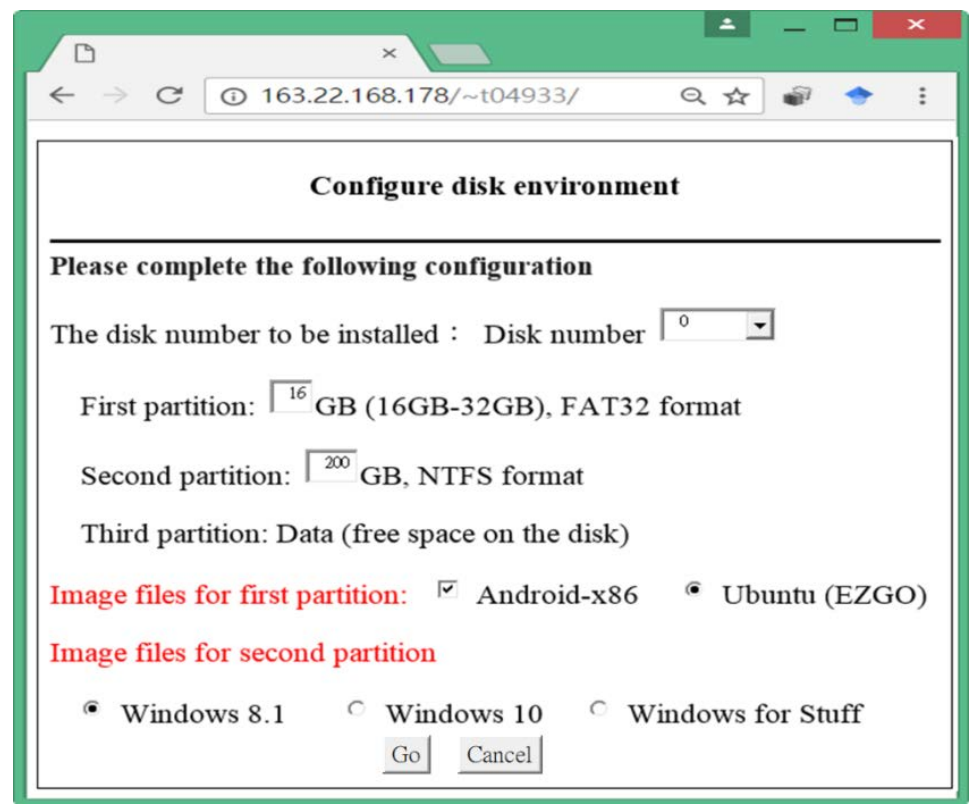

Fig. 2. Disk pre-configure service.

2) Network examination: Customized iPXE preloaded on the USB drive, booting with USB drive (see Fig. 1). Default menu will start-up iPXE, got IP from local DHCP switch, connect to enc.cyc.edu.tw HTTP server (see Fig. 3), and make a three-tier Internet connection speed check from school, ENCCYC, CCU, and TANet backbone (school-CNC-RNC-TANet, see Fig. 4).

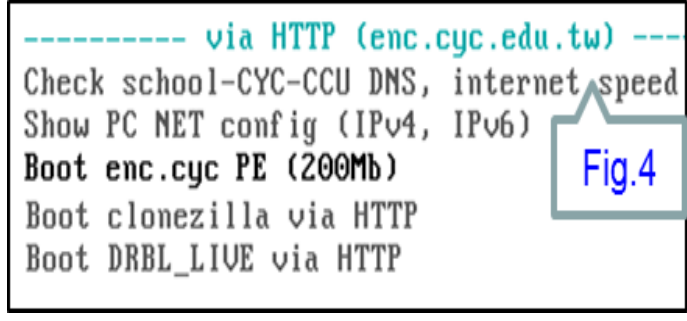

Fig. 3. The iPXE remote menu via HTTP.

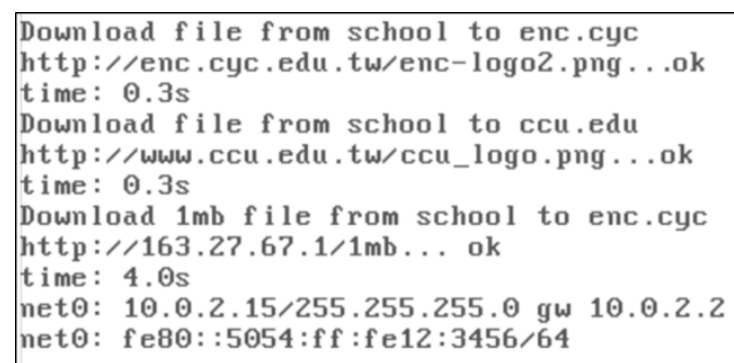

Fig. 4. Internet connection and speed test.

3) To boot iPXE WIMBoot via HTTP and multicast files: To multicast multiple files, boot iPXE WIMBoot via HTTP, run Kazilla service to synchronize files (see Fig. 5).

\subsection{Agile Infrastructure}

The mechanism is furnished with the requirements of infrastructure more agile driving force. For 
educational infrastructure, multi-tenant, integration, better utilization, and virtualization require an agiler to maximize benefit.

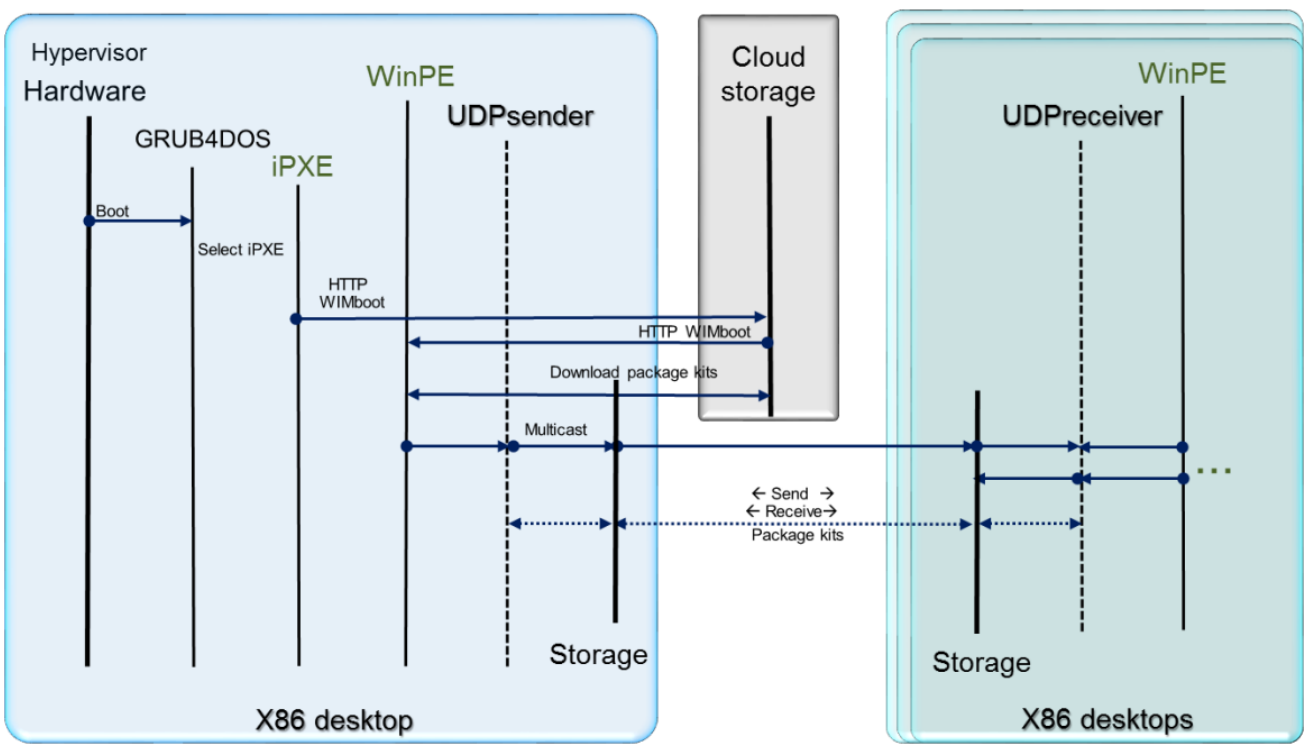

Fig. 5. The iPXE microservice flow.

\section{Related Work}

\subsection{Cloud-Native}

Cloud-Native proposed by Matt Stine [1] is a collection of Cloud technologies and enterprise management methods. It includes DevOps, Continuous Delivery, Microservices, Agile Infrastructure, and Conway's Law, as well as reorganizing companies based on commercial capabilities. The technical part of Cloud-Native is built on traditional Cloud (IaaS、PaaS、SaaS) concepts, but emphasize the method of enterprise management. The flexible infrastructure corresponds to the IaaS component, and the micro-services can correspond to PaaS and SaaS services (see Fig. 6).

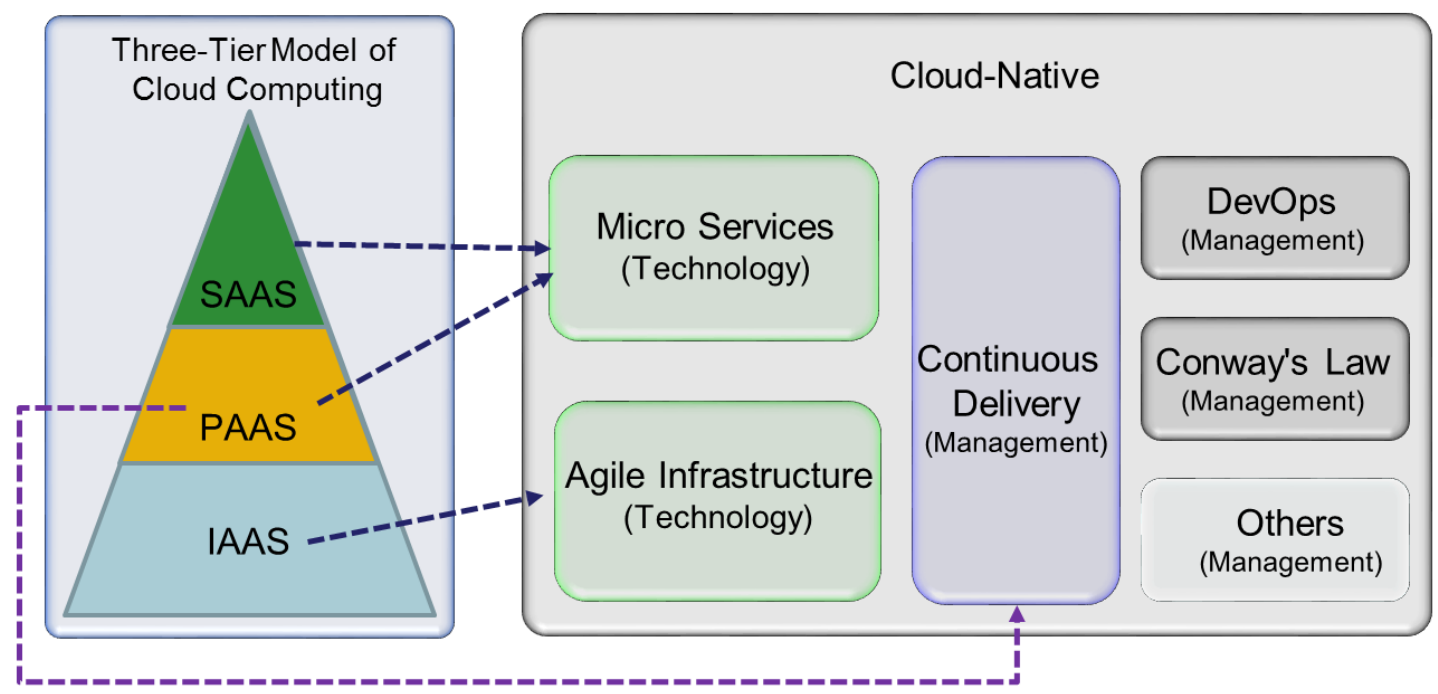

Fig. 6. Cloud computing and cloud-native.

\subsection{Virtual Disk Layering}


Desktop virtualization can be effective either server-based or client-hosted virtualization. [2] Regardless client/server virtualization, the user still need a desktop environment to be encapsulated into virtual machines. The virtual desktop infrastructure (VDI) may be the best known centralized model. VDI is a shadow copy of the entire desktop, which is stored and executed as remote virtual machine onto centralized server farm. There are many advantages use VDI [3], [4], but application performance and centralized issues retarded server side virtualization [5].

Intel proposed a different virtual desktop solution named the Intelligent Desktop Virtualization (IDV) [6]. IDV is designed to supply and maintain the client virtual images. After the images are downloaded and updated or backup with block level changes, the clients connected to the server to initialize the image. The main difference between the IDV and the VDI architecture is that IDV is executed in the real PC instead of the virtual machine. Although IDV efficiency is not heavily depending on network bandwidth, it still needs to synchronize with server frequently.

A desktop hypervisor like bare-metal or native virtualization could run multiple operating systems by hosting desktop images. Linux has supported system prevalence for a long time ago. Microsoft Hyper-V is a native desktop virtualization solution [7]. Comparing installed OS and applications on a physical disk, virtual VHDX disks are easier to satisfy layering desktop [8].

\subsection{Mass Deployment}

The system [9], described is a Linux-Windows dual-boot system, which is targeting to construct a high performance PC (HPC) cluster, in additional to equip with improved boot method which is also used in local desktop virtualization. Clonezilla is a suite of open source toolkits which simplified cloning and deployment Windows and Linux that works with Linux distributions [10], [11]. It can do 1 to N-point multicast data transmission efficiently and flexibly. Compare four cloning system: Norton Ghost, Frisbee, System Imager, and Clonezilla toolkits, created Grimiore, a web-based frontend for Clonezilla to maintain academic computer labs [12]. The iPXE work standalone accompanied by GRUB4DOS and DHCP, iPXE is a better solution to replace PXE Service [13]. UDPcast is used in multicast deployment files [14].

\section{Conclusion}

In this work, we propose the virtual desktop laying and deployment mechanism, implementing them by open source solutions. The mechanism improves the basic operating principles without additional software needs. In addition, the Cloud-Native elaborates the management and technology theme of the mechanism.

The mechanism turns educational infrastructure into agile infrastructure. The developers can continuously deliver package kit to renew environment in cloud storage. Schools applying this mechanism can recover PC handily and deploys PC rooms within hours.

The customized environment with the mechanism had been pre-installed in the computer classrooms of the Primary and Secondary Public School of Chiayi County since 2013. A total of 8,500 sets of computers had been installed as standard equipment in 165 computer classrooms and learning corner of which about $60 \%$ belong to the rural schools. Moreover, extend to six neighboring counties, about 30,000 of computers in Taiwan.

In the future release, version control will be added for software updates management. Furthermore, possible enhancements in the future have been planned: Components by DevOps method proceed remote semi-auto or auto mass deployment and updates maintenance.

\section{References}

[1] Stine, M. (2015). Migrating to cloud-native application architectures. O'Reilly Media Sebastopol. 
[2] Miller, K., \& Pegah, M. (2007, October). Virtualization: Virtually at the desktop. Proceedings of the 35th annual ACM, SIGUCCS fall conference (pp. 255-260). ACM.

[3] Petrović, T., \& Fertalj, K. (2009, October). Demystifying desktop virtualization. Proceedings of the 9th WSEAS International Conference on Applied Computer Science (pp. 241-246). World Scientific and Engineering Academy and Society (WSEAS).

[4] Lunsford, D. L. (2009). Virtualization technologies in information systems education. Journal of Information Systems Education, 20(3), 339.

[5] Dasilva, D. A., Liu, L., Bessis, N., \& Zhan, Y. (2012, October). Enabling green it through building a virtual desktop infrastructure. Proceedings of 2012 Eighth International Conference on Semantics, Knowledge and Grids (SKG) (pp. 32-38). IEEE.

[6] (2016). Retrieved

from the

website: http://www.intel.com/content/dam/doc/white-paper/intelligent-desktop-virtualization-overview-pa per.pdf

[7] Hyper-V

Overview.

(2016).

Retrieved

from

the

website: https://technet.microsoft.com/en-us/library/hh831531.aspx

[8] Download VHDX Format Specification v1.00 from Official Microsoft Download Center. (2016). Retrieved from the website: http://www.microsoft.com/en-us/download/details.aspx?id=34750

[9] Liang, S., Holmes, V., \& Kureshi, I. (2012). Hybrid computer cluster with high flexibility.

[10] Clonezilla. (2016). Clonezilla.nchc.org.tw. Retrieved from the website: http://clonezilla.nchc.org.tw/

[11] Bowling, J. (2011). Clonezilla: Build, clone, repeat. Linux journal, 011(201), 6.

[12] Meek, J. A., \& Bradshaw, M. K. (2009, October). Web based computer lab imaging with grimiore. Proceedings of the 37th Annual ACM SIGUCCS Fall Conference: Communication and Collaboration (pp. 19-24). ACM.

[13] iPXE - open source boot firmware. (2016). Ipxe.org. Retrieved from the website: http://ipxe.org/

[14] Paul, D., et al. (September 2008). Using udpcast to IP multicast data over amateur packet radio networks. Proceedings of the 27th ARRL and TAPR Digital Communications Conference.

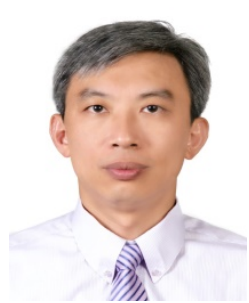

Ming-Hsun Yang is currently studying a Ph.D. at the Department of Computer Science and Information Science, National Chi Nan University, Puli, Taiwan. His research focuses on the topic of technology in education. He received his master of education in knowledge management from National Chi Nan University, Taiwan. He is a senior teacher in Education Network Center of Chiayi County, Taiwan now. His future research interest is big data and ICT in education.

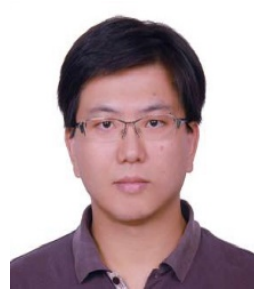

Shian-Hua Lin received his B.S., M.S. and a Ph.D. degree in engineering science from National Cheng-Kung University, Tainan, Taiwan, in 1992, 1994, and 2000, respectively. He is major in computer science. His research interests include web information retrieval, extraction, and classification, machine learning, database and knowledge management system, and web social network and digital learning systems. He is an associate professor of the Department of Computer Science and Information Science, National Chi Nan

University now. 